\title{
A investigação da cegueira noturna no grupo materno-infantil: uma revisão histórica ${ }^{1}$
}

\author{
The investigation of night blindness in the \\ mother-child group: a historical review
}

\author{
Cláudia SAUNDERS² \\ Andréa RAMALHO² \\ Patricia de Carvalho PADILHA 3,4 \\ Cristiane Chagas BARBOSA 4,5 \\ Maria do Carmo LEAL ${ }^{6}$
}

Objetivou-se neste estudo apresentar uma revisão histórica sobre a cegueira noturna, um indicador funcional da deficiência de vitamina A. Realizou-se uma revisão da literatura científica publicada no período de 1929 a 2005, nas bases de dados Medline, Lilacs, SciELO e dos comitês nacionais e internacionais de saúde e de micronutrientes, utilizando-se os termos night blindness, xerophthalmia, pregnancy, post partum women, newborn, children, vitamin A deficiency, micronutrient, deficiência de vitamina A, micronutriente, cegueira noturna, gestação, xeroftalmia. O reconhecimento da importância do indicador funcional da deficiência de vitamina A foi consagrado em 1996 pela Organização Mundial de Saúde, a partir da recomendação de uma entrevista padronizada para investigação da cegueira noturna. 0 método se caracteriza por apresentar facilidades operacionais, ser de baixo custo, além de permitir a sua aplicação tanto para o monitoramento do estado nutricional de pessoas e comunidades, quanto para a avaliação da eficácia de programas de intervenção. Entre gestantes e nutrizes, estudos recentes sugerem a utilidade deste indicador no diagnóstico dos casos de deficiência de vitamina A ainda no estágio subclínico da deficiência, sendo grande a sua concordância com indicadores bioquímicos. Ao longo dos anos, verifica-se também o reconhecimento deste indicador como um instrumento importante para a predição do risco de morbimortalidade no grupo materno-infantil.

Termos de indexação: cegueira noturna; xeroftalmia; gestação; deficiência de vitamina A.

\footnotetext{
${ }^{1}$ Artigo elaborado a partir da tese de C.S.P. COELHO, intitulada "Deficiência de vitamina A no binômio mãe-filho e distribuição intraplacentária de retinol". Escola Nacional de Saúde Pública, Fundação Oswaldo Cruz. Rio de Janeiro; 2003.

2 Universidade Federal do Rio de Janeiro, Centro de Ciências da Saúde, Departamento de Nutrição e Dietética. Av. Trompovsky s/n., Edifício dos Institutos, Bloco J, 2ํandar, Ilha do Fundão, 21944-970, Rio de Janeiro, RJ, Brasil. Correspondência para/ Correspondence to: C. SAUNDERS. E-mails: <claudiasaunders@nutricao.ufrj.br>; <claudiasaunders@osite.com.br>; <csaunders@ig.com.br>.

3 Hospital Universitário Clementino Fraga Filho. Rio de Janeiro, RJ, Brasil.

4 Universidade Federal do Rio de Janeiro. Instituto de Nutrição J osé de Castro, Programa de Pós-graduação em N utrição. Rio de Janeiro, RJ, Brasil.

${ }^{5}$ Hospital Municipal São Francisco Xavier. Rio de Janeiro, RJ, Brasil.

${ }^{6}$ Fundação Oswaldo Cruz, Escola Nacional de Saúde Pública, Departamento de Epidemiologia e Métodos Quantitativos em Saúde. Rio de Janeiro, RJ, Brasil.
} 


\section{A B S T R A C T}

The aim of this study was to provide a historical review of night blindness, a functional indicator of vitamin A deficiency. The scientific literature published in the period from 1929 to 2005 was reviewed, using Medline, Lilacs, SciELO, National and International Health and Micronutrients Comities databases. The key words used were: night blindness, xerophthalmia, pregnancy, postpartum women, newborn, children, vitamin A deficiency, micronutrient. The importance of the functional indicator of vitamin A deficiency has been consecrated in 1996 by the World Health Organization through the recommendation of a standardized interview to investigate cases of night blindness. The method is characterized by methodological easiness, low cost and it allows the monitoring of the nutritional status of people and communities, and evaluation of the efficacy of interventions. Recent studies suggest that this indicator is also useful for diagnosing subclinical vitamin A deficiency among pregnant women and postpartum women, since the agreement with biochemical indicators is expressive. Along the years, this indicator has also been recognized as an important instrument to predict the risk of morbidity and mortality in the mother-child dyad.

Indexing terms: night blindness; xerophthalmia; pregnancy; vitamin A deficiency.

\section{N T R O D U ÇÃ O}

A Organização Pan-Americana da Saúde ${ }^{1}$ declara que a deficiência de vitamina A (DVA) ainda é considerada um problema de saúde pública em países em desenvolvimento, apresentando maior prevalência entre crianças em idade pré-escolar (idade inferior a cinco anos), recém-nascidos, mulheres grávidas e nutrizes. Além de ser a causa mais importante de cegueira entre as crianças, também contribui significativamente para 0 aumento das taxas de morbi-mortalidade associadas aos processos infecciosos comuns na infância ${ }^{2-7}$, ainda que como carência subclínica ou marginal. Define-se como carência subclínica "aquela na qual as concentrações de vitamina $\mathrm{A}$ nos tecidos são suficientemente baixas para produzir conseqüências adversas para a saúde mesmo que não estejam presentes sinais de xeroftalmia" 2 .

Diante do impacto da DVA na saúde, especialmente no grupo materno-infantil, é fundamental a detecção da deficiência nos indivíduos ou comunidades em risco, sobretudo, por meio de indicadores confiáveis e que sejam capazes de retratar, com fidedignidade, a situação de saúde e nutrição do grupo estudado. Alguns dos atributos desejáveis para os indicadores são: validade e confiabilidade, capacidade para diagnóstico precoce (deficiência subclínica), simplicidade metodológica, boa cobertura, representatividade, aceitabilidade, baixo custo, e que seja facilmente incorporável às rotinas de saúde, principalmente quando os recursos são escassos $^{2,8-13}$. Os indicadores nutricionais são fundamentais para a decisão sobre quando os programas de intervenção devem ser iniciados, mantidos, modificados ou concluídos na populaçãa 2,13 .

Tendo em vista a magnitude da DVA, revelada pelas estatísticas mundiais atualizadas ${ }^{10}$, The International Vitamin A Consultative Group (IVACG) revisou recentemente, os progressos alcançados nos últimos 25 anos no controle da DVA. A revisão e a validação dos métodos diagnósticos e indicadores da DVA foram consideradas um tema prioritário ${ }^{1,9,14,15}$. Os indicadores classicamente empregados para expressar a DVA são os indicadores biológicos - funcional, bioquímico, histológico, clínico - e de grupos populacionais, denominados pela Organização Mundial de Saúde (OMS) de ecológicos ${ }^{2,13}$.

$\mathrm{Na}$ avaliação clínica, investigam-se as alterações oculares, designadas de xeroftalmia. 0 termo xeroftalmia é empregado para designar o espectro de sintomas e sinais oculares atribuídos à DVA, cujas manifestações são evolutivas e podem resultar em cegueira nutricional, muitas vezes irreversível. A xeroftalmia inclui alterações funcionais e morfológicas, apresentadas no Quadro 19,13,16. 
Quadro 1. Alterações funcional e clínicas da xeroftlamia (só tem uma alteração funcional citada no quadro que é a cegueira noturna).

\begin{tabular}{llcl}
\hline Sigla & \multicolumn{1}{c}{ Alteração } & Sigla & \multicolumn{1}{c}{ Alteração } \\
\hline XN & cegueira noturna & X3A e X3B & queratomalácia \\
X1A & xerose da conjuntiva & XS & cicatriz corneal (XS) \\
X1B & mancha de Bitot & XF & fundus xeroftalmicus \\
X2 & xerose corneal & & \\
\hline
\end{tabular}

Fontes: Sommer \& Davidson ${ }^{9}$, M cLaren \& Frigg ${ }^{13}$, Sommer ${ }^{16}$.

Nesse contexto, o indicador cegueira noturna $(X N)$ que reflete a primeira manifestação funcional da DVA, e o prejuízo na visão com pouca luz ou durante a noite, tem sido validado e amplamente empregado em estudos populacionais, sobretudo no grupo materno-infantil, sendo descritas altas prevalências de XN nesta população, considerada de maior vulnerabilidade para DVA ${ }^{2,13}$.

Este trabalho consiste em uma revisão do conhecimento disponível na literatura sobre o indicador funcional da DVA, XN. Tem como objetivo apresentar uma descrição histórica do reconhecimento deste indicador, como um instrumento promissor na investigação da DVA no grupo materno-infantil, descrever as prevalências referentes ao grupo materno-infantil, além de ressaltar sua importância diante da facilidade de incorporação à rotina dos serviços de saúde, pelas suas vantagens metodológicas.

Utilizaram-se as bases Medline, Lilacs, SciELO, dos comitês nacionais e internacionais de saúde e micronutrientes, buscando-se publicações disponíveis no período de 1929 a 2005, adotando-se os termos night blindness, xerophthalmia, pregnancy, post partum women, newborn, children, vitamin A deficiency, micronutrient, deficiência de vitamina $A$, cegueira noturna, gestação, xeroftalmia, micronutriente. Foram incluídos todos os estudos encontrados que envolviam seres humanos e estudos experimentais (envolvendo animais) publicados em inglês, português, espanhol e excluídos os trabalhos publicados em outras línguas ou disponíveis somente no formato abstracts.

\section{EPIDE M IO LOG IA DA CEGUEIRA NOTURNA NO GRUPO MATERN O-INFA N T IL}

Em uma revisão publicada recentemente ${ }^{17}$, constatou-se que, até o final da década de 1930, vários trabalhos publicados, entre esses alguns experimentais, ajudaram a elucidar o papel da vitamina A no ciclo visual. Com isso, a xeroftalmia começou a ser investigada em várias partes do mundo ${ }^{18-20}$, e os estudos vêm, sistematicamente, mostrando que tal sintoma ocular está fortemente associado com intercorrências gestacionais e maiores taxas de morbi-mortalidade materna e infantil ${ }^{3,5}$.

As regiões de maior prevalência de inadequação do estado nutricional de vitamina $\mathrm{A}$ no grupo materno infantil, são: Sul e Sudeste da Ásia, os países da África, a região Oeste do Pacífico, e a região Leste do Mediterrâneo. $\mathrm{Na}$ região das Américas encontram-se $4 \%$ dos casos, e no Brasil estima-se que 167,2 mil mulheres tenham DVA ${ }^{10}$.

Na população mundial de pré-escolares, estima-se, que, aproximadamente, 127 milhões apresentem DVA (níveis séricos de retinol $<0,7 \mu \mathrm{mol} / \mathrm{L}$ ) e que 4,4 milhões apresentem xeroftalmia. As mais altas taxas de prevalência de inadequação de retinol sérico neste grupo são registradas no Sul e Sudeste da Ásia e na África. $\mathrm{Na}$ região das Américas, temos 8,2 milhões de crianças acometidas, merecendo destaque o Brasil, onde é estimado que se concentre cerca de $30 \%$ dos casos de xeroftalmia desta região ${ }^{10}$.

Em relação ao indicador funcional, 6,2 milhões $(5,8 \%)$ das mulheres no mundo desenvolvem XN gestacional e $63,0 \%$ dos casos ocorrem anualmente no Sul e Sudeste da Ásia. Na região das Américas, calcula-se que cerca de $6,0 \%$ das mulheres são acometidas. Não existem dados de âmbito nacional sobre a prevalência da XN gestacional no Brasil. Estima-se, segundo dados baseados em estudos realizados com pré-escolares no Nordeste (a região economicamente menos desenvolvida do País), publicados pela OMS, que 
o país contribua com $33,2 \%$ da prevalência prevista para a região das Américas ${ }^{10,21}$.

Como os pré-escolares são tradicionalmente considerados como grupo de maior risco para a DVA, representa o foco prioritário das medidas intervencionais ao longo dos tempos. Conseqüentemente ao longo dos anos verifica-se uma redução significativa do número de crianças acometidas pela carência ${ }^{10,21}$. Entretanto, a investigação da DVA em mulheres em idade fértil, especialmente gestantes e nutrizes, por meio dos indicadores funcional (XN) e bioquímico (retinol sérico $<1,05 \mu \mathrm{mol} / \mathrm{L}$ ), têm revelado altas prevalências nas diversas regiões do mundo (Tabela 1) 3,8,10,14,22-24.

As novas estimativas de prevalência, com base no indicador funcional, sugerem que existam em torno $50 \%$ mais casos de XN materna do que casos de xeroftalmia em pré-escolares no mundo, apontando o sucesso no controle da DVA infantil e a relativa negligência da carência materna ${ }^{10}$. Assim, as gestantes, que são acometidas, principalmente, durante o segundo e o terceiro trimes-

Tabela 1. Prevalência mundial de XN em pré-escolares e na gestação.

\begin{tabular}{lcc}
\hline \multirow{2}{*}{ Região/País } & \multicolumn{2}{c}{ XN } \\
\cline { 2 - 3 } & Pré-escolares (\%) & Materna (\%) \\
\hline África & 1,53 & 4,4 \\
Etiópia & 4,8 & 16,0 \\
Quênia & 2 & 2,2 \\
África do Sul & 1,6 & 2,2 \\
Cambodia & 1,14 & 4,8 \\
Zâmbia & 6,2 & 11,6 \\
Leste do Mediterrâneo & 0,85 & 3,2 \\
Egito & 0,32 & 9,4 \\
Marrocos & 0,16 & 2,2 \\
Sul/Sudeste Asiático & 1,2 & 10,9 \\
Bangladesh & $0,62-1,05$ & $6,8-12,8$ \\
Índia & $1,1-1,56$ & 12,1 \\
Nepal & $0,6-3,3$ & $16,7-18,0$ \\
Oeste do Pacífico & 0,18 & 1,9 \\
Filipinas & $0,4-0,7$ & 8,6 \\
Américas & 0,16 & 3,8 \\
Peru & -- & 3,7 \\
Brasil & 0,13 &
\end{tabular}

Fontes: West et al. ${ }^{10}$, Christian $^{14}$, WHO$^{21}$ tres, passam a ser consideradas, do mesmo modo que os pré-escolares, como o grupo de maior risco para a DVA. Nas regiões onde é descrita alta prevalência de xeroftalmia em pré-escolares, a carência materna também pode ser um problema de saúde pública ${ }^{10,14,25}$.

Christian et al. ${ }^{3}$, em um estudo de base populacional, no Nepal, encontraram um risco aumentado para DVA em gestantes com XN, quando comparados aos níveis de retinol sérico inferiores a 0,70 $\mu \mathrm{mol} / \mathrm{L}(\mathrm{OR}$ - Odds ratio) 4,00) e $<1,05 \mu \mathrm{mol} / \mathrm{L}(\mathrm{OR} 2,5)$, respectivamente. Tal sintoma ocular foi associado a complicações como infecção ginecológica, diarréia, síndromes hipertensivas, náuseas e vômitos, inapetência e anemia.

A situação registrada no Rio de Janeiro, pelo Grupo de Pesquisa em Vitamina A (GPVA) do Instituto de Nutrição Josué de Castro, da Universidade Federal do Rio de Janeiro (INJC/UFRJ), vem confirmar que a XN também é um importante problema nutricional na região. Em estudo realizado na Maternidade Escola da Universidade Federal do Rio de Janeiro, encontrou-se uma prevalência de XN gestacional de $17,9 \%$, e evidenciou-se a associação obtida entre o sintoma ocular e o indicador bioquímico ${ }^{26,27}$. Verificou-se, ainda, que o menor número de consultas da assistência pré-natal e história de aborto foram as características maternas associadas com a XN gestacional. Confirmou-se ser a XN gestacional de origem nutricional nesse grupo estudado, pois, $46,2 \%$ dos casos foram observados em mulheres com níveis de retinol sérico menor que $1,05 \mu \mathrm{mol} / \mathrm{L}$ e, destas, 38,5\% apresentaram níveis entre $0,7 \mu \mathrm{mol} / \mathrm{L}$ e $1,05 \mu \mathrm{mol} / \mathrm{L}$, sugerindo que 0 indicador pode ser útil para diagnosticar os casos de DVA ainda no estágio subclínico da deficiência ${ }^{27}$.

Ramalho et al. ${ }^{28}$ descreveram um caso de xeroftalmia (ulceração corneal bilateral) em criança de cinco anos, no município do Rio de Janeiro, cujo diagnóstico da DVA foi realizado tardiamente e, com isso, houve perda de visão de um dos olhos. Os autores ressaltam que a criança perdeu a visão, não por falta de assistência, mas porque a equipe 
de saúde não considerou a deficiência de vitamina A como hipótese diagnóstica. Há motivos para supor que casos como este não são demasiadamente raros na América Latina.

$\mathrm{Na}$ Itália, Fiore et al. ${ }^{29}$ relataram o caso de um menino de 12 anos com perda da acuidade visual, apresentando XN e mancha de Bitot, em decorrência da deficiência crônica de vitamina A, ocasionada pela imposição familiar à restrição dietética desnecessária por uma suposta alergia alimentar, com melhora após a suplementação.

Considerando-se a etiologia da DVA, existem evidências de que renda e escolaridade não parecem ser os únicos fatores determinantes desta carência nutricional. Tal constatação reforça a tese de que a ingestão inadequada de alimentos fonte de vitamina A seja um importante fator etiológico da carência, e que sua exclusão ou baixo consumo possam estar mais relacionados a hábitos alimentares inadequados do que aos fatores econômicos. Assim, restrições alimentares severas e inadequadas podem causar deficiências nutricionais importantes, sobretudo de vitamina A, com risco de danos irreversíveis ao organismo ${ }^{29,30}$.

\section{O papel da vitamina A no ciclo visual}

A vitamina A tem um papel fundamental na manutenção da integridade dos processos visuais, visto que estado inadequado desta constitui a principal causa de cegueira evitável na infância ${ }^{31}$.

Na retina há dois tipos de células fotoreceptoras: cones - responsáveis pelo sentido da cor e pela visão na luz brilhante - e os bastonetes responsáveis pela acuidade visual à baixa luminosidade. Nos bastonetes, temos o pigmento retiniano - rodopsina, que é uma proteína conjugada. A reação fotoquímica da visão tem início quando o estímulo luminoso atinge a retina, a rodopsina é cindida em seu componente protéico - a opsina, e no componente não-protéico - o retinal. $\mathrm{Na}$ presença da luz, ocorrem alterações na configuração do retinal, que consiste na conversão de 11- cis-retinal a all-trans-retinal, que são acompanhadas por uma mudança global da molé- cula de rodopsina. Tais alterações funcionam como estímulo molecular para um impulso nas terminações do nervo óptico, que é transmitido ao cérebro, propiciando a visão com pouca luz. Nos casos de DVA, a síntese de rodopsina pode estar prejudicada, devido à falta da substância precursora, podendo ocorrer então a $X^{6,16,17}$, que caracteriza primeiro estágio da síndrome xeroftálmica, uma vez que a rodopsina requer altas concentrações de 11- cis-retinal para exercer a adaptação da visão com pouca luminosidade $\mathrm{e}^{12,17,31}$.

Quando a depleção das reservas de vitamina A é suficiente para afetar a função visual, um exame clínico acurado poderia evidenciá-la, embora tardiamente em relação à história natural da doença. A XN constitui-se na primeira manifestação ocular que expressa a diminuição da capacidade orgânica em regenerar a rodopsina, visto a essencialidade da vitamina $A$ à visão em baixa luminosidade. 0 impacto da DVA no ciclo visual pode ser notado por alterações em três estruturas oculares mais expressivamente: retina, conjuntiva e córnea, tendo como conseqüência desde casos de XN até cegueira parcial ou total ${ }^{31}$. O comprometimento da retina pode se dar tanto em decorrência das alterações bioquímicas/ funcionais $(\mathrm{XN})$, quanto estruturais (XF, Quadro 1 ).

0 estágio X1B, caracterizado pela mancha de Bitot, é decorrente de xerose intensa na conjuntiva, resultante do acúmulo de células epiteliais descamadas, fosfolipídeos das glândulas de Meibômio e bacilos saprófitas (corynebacterium xerosis). As alterações na córnea são, inicialmente, detectadas por declínio na produção de muco, expresso pelo sinal clínico de $X 2$, podendo conduzir à ceratomalácia, (X3A; X3B), por formação de úlcera corneal favorecendo a invasão microbiana e, por fim, cegueira parcial ou total| ${ }^{16,31}$.

\section{ACHADOS HISTÓRICOS DA CEGUEIRA NOTURNA NO GRU PO MATERNO-INFA NTIL}

Os primeiros relatos de $\mathrm{XN}$, também descrita como nictalopia e hemeralopia, datam de 
1500 a.C. no Egito, e foi a primeira doença carencial a ser claramente definida ${ }^{17,32}$. O papyrus Eberes (1600 a.C.), o mais antigo texto médico conhecido no ocidente, apresentava como terapêutica dos casos de hemeralopia a utilização de fígado cru, que seria recomendada também por Hipócrates (460-325 a.C. . 17,31.

No início do século XIX, Sepoys, na Índia, associou a cegueira noturna a uma dieta com pouca variedade de alimentos, porém foi ao longo desse século que esta investigação tornou-se mais sistemática, com a realização de experimentos durante uma expedição científica conduzida por Eduard Schawarz, entre 1857 e 1859. Nas longas viagens marítimas casos de $\mathrm{XN}$ eram notificados, e os indivíduos acometidos tratados com fígado bovino, identificando-se a XN como uma doença nutricional. A relação entre a dieta e a saúde ocular foi melhor compreendida no século seguinte, ressaltando o papel da vitamina $A$ e seu impacto na saúde pública ${ }^{33}$.

Acredita-se, entretanto, que o primeiro relato científico de lesões oculares e a possível origem da xeroftalmia decorreram da descrição de doença ocular em crianças escravas do Rio de Janeiro, pelo médico Manoel da Gama Lobo, em $1864^{31}$.

Em 1913, Osborne \& M endel identificaram a vitamina $A$, que foi inicialmente relacionada ao crescimento, em trabalhos experimentais com ratos, e mais tarde, como tratamento para $X^{17,31}$.

No Brasil, Castro ${ }^{34}$, no clássico Geografia da Fome, apresentava o problema da fome oculta, carência de diversas vitaminas, incluindo a de vitamina A. 0 autor descreveu que a XN era comum no início do século $X X$, principalmente em crianças do sertão nordestino, e apontava a alimentação deficiente como a causa principal.

Outros casos de hemeralopia, a falsa cegueira que ocorria durante as secas, também foram citados por Diniz \& Santos ${ }^{31}$ nas áreas mais pobres do País. Porém, não se encontrou referência a casos de XN durante a gestação, ou lactação, ou em mulheres na idade reprodutiva, até a presente data, e nenhum termo regional foi encontrado para designar o sintoma ocular.

A XN durante a gestação associada à DVA é destacada, inicialmente, na literatura a partir de 1930 35,36. Em 1966, Dixit22 chamou a atenção da comunidade científica para a alta prevalência de XN em 203 gestantes residentes em área rural da Índia. 0 diagnóstico foi realizado com uma entrevista e foi verificada maior prevalência no terceiro trimestre gestacional. Contudo, 0 autor sugere que esse sintoma ocular pode ser fisiológico, pois constatou o seu desaparecimento espontâneo na primeira semana após o parto, porém dados recentes confrontam tais hipóteses, pois descrevem a associação entre tal achado ocular e maiores taxas de mortalidade materna ${ }^{5,37}$.

Na década de 70 , Simmons \& Melo ${ }^{38}$ e Simmons ${ }^{39}$ investigaram dados referentes à xeroftalmia e à cegueira noturna em Estados do Nordeste brasileiro, pela análise de registros de pacientes internados e ambulatoriais, reconhecendo a xeroftalmia como um problema de saúde pública na região, sendo a causa de cegueira em algumas áreas da região.

$\mathrm{O}$ indicador $\mathrm{XN}$ passou a ser estudado novamente, a partir da década de 80 . Sommer et al..$^{40}$ propuseram uma entrevista padronizada para o diagnóstico da XN, por meio de estudos realizados com 5.925 pré-escolares, na Indonésia, onde comprovou-se a validade (sensibilidade e alta especificidade) da entrevista subjetiva da XN, em relação à prova objetiva de visão noturna - visão escotópica, método diagnóstico que era inicialmente preconizado, e retinol sérico.

Para a aplicação do método visão escotópica é requerido conhecimento oftalmológico, equipamento complexo e caro, o que dificulta a aplicação em trabalhos de campo. Tal método, bem como os outros também capazes de diagnosticar a cegueira noturna, como, por exemplo, o tempo de restauração da visão ou eletrorretinografia, necessita da cooperação e do interesse do indivíduo avaliado, o que dificulta o seu emprego em crianças de pouca idade $e^{2,13,16}$. 
Desde 1996, a OMS² e a comunidade científica vêm recomendando, para diagnosticar a XN, sobretudo no grupo materno-infantil, uma entrevista padronizada (Quadro 2), que se caracteriza pela facilidade metodológica, uma vez que para a sua aplicação não se requer o uso de equipamentos caros e conhecimento oftalmológico especializado, além de apresentar boa associação com os indicadores bioquímicos, ser de baixo custo e permitir a investigação em estudos populacionais $^{14}$.

Na prática, este instrumento visa aumentar a sensibilidade e reduzir o erro de classificação da $X N$, devendo ser empregada usando-se linguagem local, a fim de identificar corretamente o termo da localidade que caracterize tal sintoma ocular na população estudada, pois o não conhecimento deste pode comprometer a fidedignidade do diagnóstico, subestimando os casos de $\mathrm{XN}^{2,13,41,42}$.

Estudos de base populacional, na Ásia, chamam atenção para as altas prevalências ( $5 \%$ a $18 \%$ ) em gestantes e nutrizes da região, a partir da investigação da XN pela entrevista, demonstrando o maior risco de morbi-mortalidade da mãe e dos lactentes nos primeiros seis meses de vida, fortemente associado à XN materna ${ }^{3-5,8,24,43,44}$. Vale ressaltar que a associação entre a $X \mathrm{~N}$ e a inadequação dos níveis séricos de retinol materno foi encontrada na maioria dos estudos citados, confirmando a causa nutricional do sintoma ocular e validando a entrevista padronizada para 0 diagnóstico da DVA materna ${ }^{3,7,8}$.

Em 2002, a XN durante a gestação passa a ser sugerida como marcador da gestação de alto risco, pois permite a identificação de gestantes

Quadro 2. Entrevista para investigação da cegueira noturna.

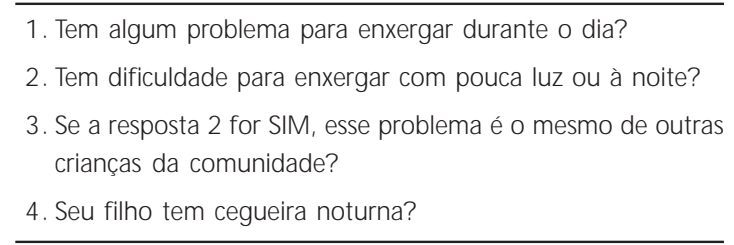
Fonte: $\mathrm{WHO}^{2}$. que necessitam de maior atenção no pré-natal, dada à maior vulnerabilidade para o desenvolvimento de complicações gestacionais, desnutrição, anemia e resultado obstétrico indesejável ${ }^{6,14}$. Uma prevalência maior ou igual a $5 \%$ de XN gestacional passa a ser sugerida para classificar a DVA como um problema de saúde pública6 ${ }^{6}$.

Poucos pesquisadores realizaram uma análise crítica sobre os aspectos relacionados à validação da entrevista padronizada para o diagnóstico da DVA em gestantes, puérperas e nutrizes. Entretanto, Saunders et al. ${ }^{26,27}$ validaram a entrevista proposta pela OM S para investigação da XN gestacional para puérperas no pós-parto imediato (até 6 horas), encontrando associação entre os níveis séricos de retinol e a prevalência de $X N$, recomendando a incorporação desta na assistência nutricional pré-natal, a fim de diagnosticar precocemente e minimizar o impacto da DVA na díade mãe-filho, favorecendo o bom resultado obstétrico.

Recentemente, Taren et al. ${ }^{25}$ publicaram um estudo, com gestantes atendidas numa maternidade pública do Nepal, visando validar o teste de visão noturna limiar (TVNL), que é um teste de adaptometria ao escuro. De acordo com os resultados, o TVNL teve grande sensibilidade $(0,73 \times 0,19)$ e baixa especificidade $(0,51 \times 0,87)$, comparando com a entrevista para XN em mulheres com níveis séricos de vitamina $A$ menores que $0,70 \mu \mathrm{mol} / \mathrm{L}$, e melhor sensibilidade $(100 \times 0,73)$ e especificidade similar $(0,51 \times 0,50)$ para mulheres com níveis séricos de vitamina $A$ menores que $0,35 \mu \mathrm{mol} / \mathrm{L}$, considerando o teste a melhor forma de classificar gestantes com concentração muito baixa de vitamina $A$. Entretanto, o trabalho em questão não utilizou a entrevista para diagnóstico de XN preconizada pela $\mathrm{OMS}^{2}$ e não considerou o ponto de corte para deficiência subclínica (abaixo de 1,05 $\mu \mathrm{mol} / \mathrm{L}$ ), que vários autores vêm apontando como o limite para apresentação de sinais de deficiência marginal de vitamina A em pré-escolares, gestantes e puérperas ${ }^{3,8-11}$. Com isso, pode-se sugerir que a aplicação do referido método TVNL poderia subestimar os casos de DVA subclínica. 
O indicador XN é, atualmente, reconhecido como um bom preditor da vulnerabilidade da família para a DVA, e sua investigação rotineira passa a ser recomendada em regiões onde a carência nutricional é prevalente, contribuindo para o fornecimento de subsídios para programas de intervenção, bem como avaliando o impacto das medidas intervencionais $2,6,13,45$.

\section{Medidas preventivas e intervencionais para casos de cegueira noturna}

As medidas preventivas e intervencionistas para os casos de $X N$ devem ser pautadas na suplementação com vitamina $\mathrm{A}$ (Quadro 3$)^{46}$, fortificação de alimentos, e diversificação alimentar, reforçando a importância da inclusão dos alimentos fontes de vitamina A no planejamento dietético ${ }^{6,37}$.

Haskell et al. ${ }^{47}$, observando gestantes com XN no Nepal, concluíram que a melhora na adaptação ao escuro não diferencia significativamente entre gestantes que receberam vitamina A de alimentos considerados fontes, como fígado, cenoura ou por via medicamentosa, com o palmitato de retinil.

Com esse estudo, abre-se um espaço para discussão de qual seria a melhor forma de suplementar a vitamina A: via suplemento (medicamento) por fontes alimentares. Certamente, vários questionamentos emergem ao tratar da suplementação de vitamina A para gestantes, entre os quais destacam-se o possível efeito teratogênico da hipervitaminose A. Portanto, tal hipótese tem dificultado a implementação de programas de

Quadro 3.Dose suplementação de vitamina A recomendada pelo Ministério da Saúde.

Lactentes a partir de 6 meses - $100.000 \mathrm{UI}$

Crianças maiores de 1 ano - $200.000 \mathrm{UI}$

Puérperas - 200.000 UI em dose única no pós-parto, antes da alta hospitalar.

Fonte: Brasil ${ }^{46}$. combate à carência desta vitamina, sobretudo a proposta de enriquecimento de alimentos ${ }^{48}$.

A literatura é controversa e dispõe de poucas informações sobre e efeito da suplementação em doses excessivas em gestantes, pelas questões éticas e metodológicas envolvidas na realização de estudos prospectivos administrando às gestantes doses suscetíveis de ter efeitos teratogênicos. Estudos científicos publicados sob o tema, apontam que existem evidências de que a vitamina $\mathrm{A}$ em excesso, durante as primeiras semanas de gestação, é teratogênica em humanos $^{48-50}$. É geralmente aceito que o retinol e seus metabólitos não têm efeito teratogênico em concentrações séricas fisiológicas, e que o ácido retinóico desempenha um papel essencial na regulação de numerosos aspectos da embriogênese normal. Um consumo excessivo de vitamina A pré-formada (25.000Ul/dia), parece estar associado com maior número de anomalias do aparelho urinário. Também foi demonstrado um maior risco de anomalias congênitas nos conceptos de mulheres que consomem mais de $15.000 \mathrm{UI}$ de vitamina A/dia pela alimentação, ou mais de $10.000 \mathrm{UI}$ sob a forma de suplementos $\mathrm{s}^{48,50}$.

O IOM ${ }^{49}$ descreve que o período crítico para o desenvolvimento das anomalias congênitas é 0 primeiro trimestre de gestação. Diz ainda que os defeitos primários, associados com a ingestão excessiva de vitamina $A$, são derivados das células da crista neural craniana e que as má-formações observadas são craniofaciais, e anormalidades no sistema nervoso central (exceto defeitos de tubo neural), no timo e no coração.

A maioria dos estudos disponíveis recomenda doses diárias de $10.000 \mathrm{UI}(3.000 \mu \mathrm{g}$ de retinol) ou dose semanal de $25.000 \mathrm{Ul}(7500 \mu \mathrm{g}$ de retinol), como quantidade segura para mulheres durante toda a gestação. Sabe-se que o risco de concentrações elevadas de metabólitos no sangue é menor quando a fonte de vitamina A é natural, ou seja, a partir da alimentação, do que sob a forma de suplemento. Assim, essas conclusões refletem as recomendações atuais a favor da utilização de fontes alimentares para melhoria do estado de vitamina $\mathrm{A}$ de gestantes ${ }^{48}$. 
No Brasil, o Ministério da Saúde ${ }^{46}$ recomenda a suplementação somente de mulheres no pós-parto imediato, nas áreas de risco para a deficiência (Nordeste, regiões de M inas Gerais, e Vale do Ribeira em São Paulo), devido ao maior risco de má-formação congênita em casos de ingestão excessiva do nutriente.

Considerando-se a importância da orientação nutricional, a diversificação do consumo de alimentos deve ser estimulada, incluindo tanto as fontes animais de vitamina $A$, quanto as de origem vegetal, que são fonte de beta-caroteno, um precursor da vitamina $A$ ativa, a fim de atender a recomendação dietética de referência ${ }^{49}$ (Quadro 4) para gestantes e crianças, minimizando o risco de deficiência.

Quadro 4. Recomendação de Ingestão de vitamina A para o grupo materno-infantil.

\begin{tabular}{lc}
\hline \multicolumn{1}{c}{ Grupo } & Ingestão dietética de referência para vitamina A \\
\hline Gestantes & \\
$14-18$ anos & $750 \mu \mathrm{g}$ de EAR/dia \\
$>19$ anos & $770 \mu \mathrm{g}$ de EAR/dia \\
Nutrizes & \\
$14-18$ anos & $1200 \mu \mathrm{g}$ de EAR/dia \\
$>19$ anos & $1300 \mu \mathrm{g}$ de EAR/dia \\
Crianças & \\
$0-6$ meses & $400 \mu \mathrm{g}$ de EAR/dia \\
$7-12$ meses & $500 \mu \mathrm{g}$ de EAR/dia \\
$1-3$ anos & $300 \mu \mathrm{g}$ de EAR/dia \\
$4-8$ anos & $400 \mu \mathrm{g}$ de EAR/dia \\
$9-13$ anos & $600 \mu \mathrm{g}$ de EAR/dia \\
\hline
\end{tabular}

EAR: Equivalente de atividade de retinol.

Fonte: Institute of Medicine ${ }^{49}$.

Na assistência às gestantes, os profissionais devem organizar suas rotinas, visando à prevenção de suplementação excessiva e indiscriminada de vitamina $A$, baseando também a orientação nutricional com teor de vitamina $A$ que não ultrapasse a dose segura para a faixa etária.

\section{O NSIDERA Ç Õ SS FINAIS}

O estado inadequado de vitamina A caracteriza-se como um importante problema de saúde e nutrição entre mulheres e crianças, podendo acarretar conseqüências indesejáveis para a saúde reprodutiva e repercutir negativamente no desenvolvimento infantil, sendo de grande relevância na saúde de recém-nascidos e lactentes, refletindo nos índices de morbimortalidade.

O impacto da deficiência de vitamina A na saúde reprodutiva tem sido bem documentado, em relação às repercussões na saúde materna e dos lactentes. Atendendo às expectativas dos comitês internacionais de saúde e da comunidade científica, alguns indicadores menos invasivos e de baixo custo têm sido testados e validados para o diagnóstico da DVA.

O papel da vitamina $A$ no ciclo visual é bem elucidado na literatura. Do mesmo modo, 0 reconhecimento da importância do indicador funcional da DVA é bem descrito pela comunidade científica. Tal indicador tem sido consagrado e a entrevista padronizada tem sido amplamente adotada na investigação da XN, permitindo a identificação de populações em risco dessa deficiência nutricional, e permitindo também o monitormento e a avaliação do impacto dos programas de intervenção.

Face ao exposto, a investigação de XN é um indicador promissor do estado nutricional de vitamina A no grupo materno-infantil, facilmente incorporável às rotinas de saúde, possibilitando medidas de prevenção e erradicação da DVA.

Quanto à suplementação, deve-se prevenir o uso excessivo e indiscriminado de suplementos de vitamina A em gestantes, e, quando necessária, deve seguir as recomendações preconizadas por órgãos competentes, dentro dos limites seguros para cada faixa etária. Recomenda-se a realização de orientação nutricional, com ênfase no consumo de alimentos fontes de vitamina $A$, iniciada em fases precoce do período gestacional, como forma de diagnosticar, tratar e prevenir a DVA e suas conseqüências para o binômio mãe-filho.

\section{REFERÊ N CIAS}

1. Organización Panamericana de la Salud. Visión Integrada de la suplementación con vitamina A en 
las Américas. 2-4 de mayo del 2001, Managua, Nicaragua. Informe de la Reunión Regional. HPP/ HPN/M N/49-17. Washington (DC): OPS; 2001.

2. World Health Organization. Indicators for assessing vitamin A deficiency and their application in monitoring and evaluating intervention programs. WHO/NUT/96.10. Geneva: WHO; 1996.

3. Christian P, West Jr KP, Khatry SK, Katz J, Shrestha SR, Kimbrough-Pradhan E, et al. Night blindness of pregnancy in rural Nepal: nutritional and health risks. Int J Epidemiol. 1998; 27(2):231-7.

4. Christian P, West J $\mathrm{KP}$, Khatry SK, Kimbrough-Pradhan E, Leclerq SC, Katz J, et al. Night blindness during pregnancy and subsequent mortality among women in Nepal: Effects of vitamin A and $\beta$-carotene supplementation. Am J Epidemiol. 2000; 152(6):542-7.

5. Christian P, West Jr KP, Khatry SK, Katz J, Leclerq SC, Kimbrough-Pradhan $E$, et al. Maternal night blindness increases risk of mortality in the first 6 months of life among infants in Nepal. J Nut. 2001; 131(5):1510-2.

6. International Vitamin A Consultative Group. Statement. Maternal night blindness: a new indicator of vitamin A deficiency. Washington (DC): IVACG; 2002.

7. Radhika MS, Bhaskaram P, Balakrishna N, Ramalakshmi BA, Devi S, Kumar BS. Effects of vitamin A deficiency during pregnancy on maternal and child health. BJOG. 2002; 109(6):689-93.

8. Biswas AB, M itra NK, Chakraborty I, Basu S, Kumar $\mathrm{S}$. Evaluation of vitamin A status during pregnancy. J Indian Med Assoc. 2000; 98(9):525-9.

9. Sommer A, Davidson FR. Assessment and control of vitamin A deficiency: the annecy accords. J Nutr. 2002; 132(9 Suppl):2845S-50S

10. West Jr KP. Extent of vitamin A deficiency among preschool children and women of reproductive age. J Nutr. 2002; 132(9 Suppl):2857S-66S.

11. Wondmikun Y. Dark adaptation pattern of pregnant women as an indicator of functional disturbance at acceptable serum vitamin A levels. Eur J Clin Nutr. 2002; 56(5):462-6.

12. Underw ood B. M ethods for assessment of vitamin A status. J Nutr. 1990; 120 (11 Suppl):1459S-63S.

13. M cLaren DS, Frigg M. Manual de ver y vivir sobre los transtornos por deficiencia de vitamina $A$ (VADD). Washington (DC): OPS; 1999.

14. Christian P. Recommendations for indicators: night blindness during pregnancy - a simple tool to assess vitamin A deficiency in a population. J Nutr. 2002; 132 (9 Suppl): 2884S-8S.
15. Ramakrishnan U, Darnton-Hill I. Assessment and control of vitamin A deficiency disorders. J Nutr. 2002; 132(9 Suppl):2947S-53S.

16. Sommer A. La carencia de vitamina A y sus consecuencias. Guía práctica para la detección y el tratamiento. Ginebra: OMS; 1995.

17. Wolf $G$. The discovery of the visual function of vitamin A. J Nutr. 2001; 131(6):1647-50.

18. McLaren DS, Shirajian E, Tchalian M, Khoury G. Xerophthalmia in Jordan. Am J Clin Nutr. 1965; 17(3):117-30.

19. Oomen HAPC, Mclaren DS, Escapini H. Epidemiology and public health aspects of hypovitaminosis A. Trop Geogr Med. 1964; 16:271-315.

20. Pillat $A$. Does xeroftalmia exist in adults? Arch Ophthalmol. 1929; 2:256-87.

21. World Health Organization. Global prevalence of vitamin A deficiency. WHO/NUT/95.3. Geneva: WHO; 1995.

22. Dixit DT. Night-blindness in third trimester of pregnancy. Indian J Med Res. 1966; 54(8):791-5.

23. Katz J, West Jr KP, Khatry SK, Pradhan EK, Leclerq SC, Christian P, et al. M aternal low-dose vitamin A or bcarotene supplementation has no effect on fetal loss and early infant mortality: a randomized cluster trial in Nepal. Am J Clin Nutr. 2000; 71(6):1570-6.

24. Christian P. Micronutrients and Reproductive Health Issues: an International Perspective. J Nutr. 2003; 133(6):1969S-73S.

25. Taren DL, Duncan B, Shrestha K, Shrestha N, Genaro-Wolf $D$, Schleicher RL, et al. The night vision threshold test is a better predictor of low serum vitamin A concentration than self-reported night blindness in pregnant urban Nepalese women. J Nutr. 2004; 134(10):2573-8.

26. Saunders C, Leal MC, Gomes MM, Campos LFC, Silva BAS, Lima APPT, et al. Gestational night blindness in women treated at a public maternity hospital in Rio de Janeiro, Brazil. J Health Popul Nutr. 2004; 22(4):348-56.

27. Saunders $C$, Ramalho RA, Pereira Thiapó de Lima AP, Martins Gomes M, Ferreira Campos L, Amaral dos Santos Silva B, et al. Association between gestational night blindness and serum retinol in mother/newborn pairs in the city of Rio de Janeiro, Brazil. Nutrition. 2005; 21(4):456-61.

28. Ramalho A, Accioly E, Flores, H. Xeroftalmia no diagnosticada en Rio de Janeiro, Brasil. Rev Chil Nutr. 1999; 26(3):316-9.

29. Fiore P, De Marco R, Sacco O, Priolo E. Nightblindness, xerophtalmia, and severe loss of 
visual acuity due to unnecessary dietary restriction. Nutrition 2004; 20(5):477.

30. Ramalho RA, Saunders C, Accioly E, Paiva F, Cardoso LO, Natalizi D. A associação entre antropometria materna e o estado nutricional de vitamina $A$ materno e dos recém-nascidos. Rev Ciênc Méd. 2001; 10(1):5-10.

31. Diniz AS, Santos LMP. Hipovitaminose A e xeroftalmia. J Pediatr. 2000; 76(Suppl 3):S311-22.

32. Wolf G. A historical note on the mode of administration of vitamin A for the cure of night blindness. Am J Clin Nutr. 1978; 31(2):290-2.

33. Underwood BA. Vitamin A deficiency disorders: international efforts to control a preventable "Pox". J Nutr. 2004; 134(1):231-6.

34. Castro J. Geografia da fome. Rio de Janeiro: Casa do Estudante do Brasil; 1959.

35. Green HN, Pindar D, Davis G, M ellanby E. Diet as a prophylactic agent against puerperal sepsis. BMJ. 1931; 2:595-8.

36. Ricketts WA. Vitamin A deficiency in pregnancy. Am J Obstet Gynecol. 1939; 38(3):484-8.

37. Saunders C, Leal MC, Ramalho RA. Vitamin A. Night blindness: a promising indicator for vitamin A deficiency. The Nest. 2003; 15:6-8.

38. Simmons WK, M ello A. Blindness in the nine states of northeast, Brazil. Am J Clin Nutr. 1975; 28(3):202.

39. Simmons WK. Xerophtalmia and blindness in northeast Brazil. Am J Clin Nutr. 1976; 29(1): 116-22.

40. Sommer A, Hussaini G, M uhilal, Tarwotjo I, Susanto $D$, Saroso S. History of nightblindness: a simple tool for xerophthalmia screening. Am J Clin Nutr. 1980; 33(4):887-91.

41. Mason JB, Lotfi M, Dalmiya N, Sethuraman K, Deitchler M. The M icronutrient Report. Current progress and trends in the control of vitamin $A$, iodine, and iron deficiencies. Ottawa, Canada: UNICEF; 2001.

42. Wedner SH, Ross DA, Congdon N, Balira R, Spitzer $\mathrm{V}$, Foster $\mathrm{A}$. Validation of night blindness reports among children and women in a vitamin $A$ deficient population in rural Tanzania. Eur J Clin Nutr. 2004; 58(3):409-19.

43. Katz J, Khatry SK, West J r KP, Humphrey JH, Leclerq SC, Pradhan EK, et al. Night blindness is prevalent during pregnancy and lactation in rural Nepal. J Nutr. 1995; 125(8):2122-7.

44. West Jr KP, Katz J, Khatry SK, Leclerq SC, Pradhan EK, Shrestha SR, et al. Double blind, cluster randomised trial of low dose supplementation with vitamin $A$ or $\beta$-carotene on mortality related to pregnancy in Nepal. BMJ. 1999; 318(7183): 570-5.

45. International Vitamin A Consultative Group Statement. Maternal night blindness: extent an associated risk factors. Washington (DC): IVACG; 1997.

46. Brasil. Ministério da Saúde. Secretaria de Atenção a Saúde. Departamento de Atenção Básica. Vitamina A mais: Programa Nacional de suplementação de vitamina $A$ : condutas gerais. Brasília: Ministério da Saúde; 2004.

47. Haskell MJ, Pandey P, Graham JM, Peerson JM, Shrestha RK, Brown KH. Recovery from impaired dark adaptation in nightblind pregnant Nepali women who receive small daily doses of vitamin A as amaranth leaves, carrots, goat liver, vitamin A-fortified rice, or retinyl palmitate. Am J Clin Nutr. 2005; 81(2):461-71.

48. Chagas MHC, Flores $H$, Campos FACS, Santana RA, Lins ECB. Teratogenia da vitamina A. Rev Bras Saúde Matern Infant. 2003; 3(3):247-52.

49. Institute of Medicine. Dietary Reference Intakes for vitamin A, vitamin $\mathrm{K}$, arsenic, boron, chromium, copper, iodine, iron, manganese, molybdenum, nickel, silicon, vanadium an zinc. Washington (DC): National Academy Press; 2001.

50. Organização Mundial de Saúde. Iniciativa sobre Micronutriente. Centro Colaborador de Alimentação e Nutrição do Nordeste I. Vitamina " $A$ " na Gestação e Lactação. Recomendações e relatório de uma consultoria. Recife: OMS; 2001

Recebido em: 30/11/2005

Versão final respresentada em: 14/7/2006

Aprovado em: 3/10/2006 